\title{
A summary of methods applied to tool condition monitoring in drilling
}

\author{
Erkki Jantunen * \\ Technical Research Centre of Finland, PO Box 1702, FIN-02044 VTT, Finland
}

Received 31 May 2001; received in revised form 18 March 2002; accepted 22 March 2002

\begin{abstract}
This paper presents a summary of the monitoring methods, signal analysis and diagnostic techniques for tool wear and failure monitoring in drilling that have been tested and reported in the literature. The paper covers only indirect monitoring methods such as force, vibration and current measurements, i.e. direct monitoring methods based on dimensional measurement etc. are not included. Signal analysis techniques cover all the methods that have been used with indirect measurements including e.g. statistical parameters and Fast Fourier and Wavelet Transform. Only a limited number of automatic diagnostic tools have been developed for diagnosis of the condition of the tool in drilling. All of these rather diverse approaches that have been available are covered in this study. In the reported material there are both success stories and also those that have not been so successful. Only in a few of the papers have attempts been made to compare the chosen approach with other methods. Many of the papers only present one approach and unfortunately quite often the test material of the study is limited especially in what comes to the cutting process parameter variation, i.e. variation of cutting speed, feed rate, drill diameter and material and also workpiece material. (C) 2002 Published by Elsevier Science Ltd.
\end{abstract}

Keywords: Tool wear; Drilling; Monitoring methods; Signal analysis; Diagnostic tools

\section{Introduction}

Tool wear and failure monitoring has raised quite a lot of interest among researchers and has consequently been studied in a number of research projects by a number of research organisations. The reason for the interest is that tool condition monitoring is considered important for the following reasons:

- Unmanned production is possible only if there is a method available for tool wear monitoring and tool breakage detection.

- Tool wear influences the quality of the surface finish and the dimensions of the parts that are manufactured.

- The economical tool life cannot be benefited from without a means for tool wear monitoring because of variations in tool life.

- Today tool changes are made based on conservative

\footnotetext{
* Fax: +358-9-460627.

E-mail address: erkki.jantunen@vtt.fi (E. Jantunen).
}

estimates of tool life which does not take into account sudden failures and at the same time leads to an unnecessarily high number of changes because the full lifetime of tools is not taken into account and consequently valuable production time is lost.

- As a consequence of the above, automated production control is not really possible without a means for tool wear monitoring.

The economical values involved in modern manufacturing are very high because of the high investments in the manufacturing equipment and naturally it would be in the interest of the industry to benefit from the equipment in an optimal way including automated production with high availability.

In principle, the tool wear monitoring methods can be classified in two categories, i.e. direct and indirect methods. With direct methods it is possible to determine tool wear directly, which means that these methods really measure tool wear as such. In spite of the many attempts direct methods such as visual inspection or computer vision etc. have not yet proven to be very 
attractive economically nor technically. In this paper only indirect measuring methods such as torque or vibration are covered. In fact the paper tries to cover all the indirect methods in drilling that have been found in the literature search that has been carried out.

There are differences in how well certain monitoring methods work depending on the purpose they are used for in tool condition monitoring. Some of the methods are more effective for detection of a sudden failure and some are more suited for tool wear monitoring. In this respect there is even more distinction in the suitability of the different signal analysis methods. It could even be claimed that the most effective and reliable methods for tool wear monitoring are so slow in practise that they are not suitable for the detection of sudden failures. Again the paper tries to cover both aspects when individual analysis techniques are discussed.

Drill wear is a progressive process which takes place at the outer margin of the flutes of the drill due to the intimate contact and elevated temperatures at the tool workpiece contact [1]. However, under constant cutting conditions drill failure is a stochastic process. The reasons for varying drill life are the inhomogeneities in the workpiece and drill materials, the irregularities in the cutting fluid motion and the unavoidable asymmetry introduced during the grinding of the cutting edges.

Similarly, as in the case of measuring methods, quite a number of signal analysis techniques have been tested for tool wear monitoring. In machining there are many disturbances and even the process as such can be run using different process parameters and hence signal analysis is really needed in order to be able to separate the wanted information from the rest of the "noise".

During the recent years quite a lot of effort has been spent on developing methods for automatic diagnosis of tool wear because automation of diagnosis is also needed in order to facilitate automatic production systems. Especially different types of neural networks have gained a lot of interest. The attempts to make the diagnosis automatic are also covered in this paper.

\section{Measuring methods}

A summary of the monitoring methods that various researchers have studied is shown in Table 1. In addition to the methods that have been tested and described in each reference, the possible coverage of the effect of cutting speed and feed rate is shown in Table 1, i.e. the table shows whether the researchers have tried to cover the effect of cutting conditions to the measured signals and calculated parameters. One reason for measuring cutting speed and feed rate is the use of these as parameters in adaptive control systems, e.g. [2].

Torque, drift and feed force together with strain measurement are all measures of cutting forces and are treated together in the subsequent study. In Table 1 the reported strain measurements are tabulated in the applicable force category because strain as such is linked to the force: force transducers actually measure strain which then is transposed to force. Spindle motor and feed drive current are closely related to the forces, i.e. they too measure the same cutting forces and phenomena, although through a longer measuring chain where also other factors influence the signals. Again spindle motor and feed drive current are treated together in the subsequent studies.

Vibration, sound, ultrasonic vibration and acoustic emission are actually all vibration measurements, although the frequency range in each of these differs and, in addition to that, sound is airborne vibration when all the others are mechanical vibrations of the structure. The frequency range in vibration measurements is typically from about $1 \mathrm{~Hz}$ to about $10 \mathrm{kHz}$ (or 20 or $16 \mathrm{kHz}$ is used as a limit [3]); in sound measurements the range is from $20 \mathrm{~Hz}$ to $20 \mathrm{kHz}$, which is the range a young person can hear; in ultrasonic vibration the frequency range is from $20 \mathrm{kHz}$ to about $80 \mathrm{kHz}$ [4]; and acoustic emission starts where ultrasonic vibration ends up and ranges to about $1 \mathrm{MHz}$. Again all the vibration related techniques are treated together. In some cases the measured vibration frequencies do not fall into the limits defined above and if this is the case then both categories are marked with " $x$ ". This is the case e.g. for vibration and ultrasonic vibration which have both been marked when the band-passed frequency is from 0.5 to $40 \mathrm{kHz}$, as it is in [5].

\subsection{Torque, drift force and feed force}

It is very logical to monitor forces in a cutting process in order to follow the development of cutting tool wear. It is generally known that cutting forces increase as tool wear increases [6]. This is due to the increase of friction between tool and workpiece. In drilling it is possible to monitor torque, drift forces (lateral forces affecting the workpiece) and the feed (thrust, $z$-axis) force. All of these have been monitored in Ref. [7]. The idea behind monitoring torque and feed force is very clear, i.e. it is expected that these forces change as the tool gradually wears. The thrust force has been used as the only measured signal in $[1,8-10]$. The simultaneous monitoring of thrust force and torque is rather common (see e.g. [2,6,11-19]) and special electronics have been developed for this purpose [11].

Drill wear as such differs to some extent from the wear of other cutting tools. Due to production tolerances a drill is slightly asymmetric, therefore it only wears at one lip until the height of both lips is equal $[7,20]$. The second lip, which is now sharper, starts cutting. This alternating process continues until neither lip has no more clearance at the margin. In the end the drill sticks 
Table 1

Summary of monitoring methods that have been studied for tool condition monitoring in drilling

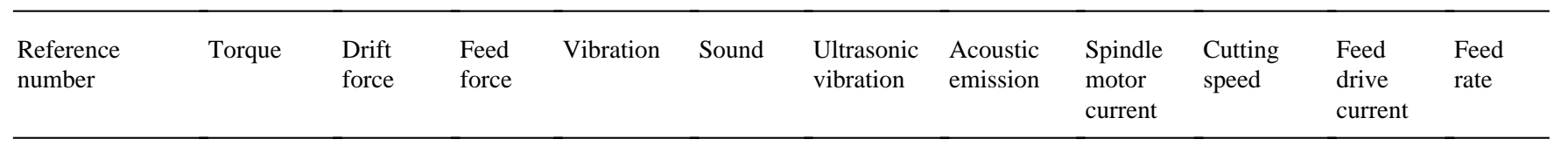

\begin{tabular}{|c|c|c|c|c|c|c|c|c|c|c|c|}
\hline [1] & & & $x$ & & & & & & $x$ & & $\times$ \\
\hline [2] & $\times$ & & $\times$ & & & & & & $\times$ & & $x$ \\
\hline [3] & $x$ & $x$ & $\times$ & $x$ & & $\times$ & & & $x$ & & $\times$ \\
\hline [4] & & & & $x$ & & $x$ & & & $x$ & & \\
\hline [5] & & & $\times$ & $x$ & & $x$ & & & $x$ & & $\times$ \\
\hline [6] & $x$ & & $\times$ & & & & & & $x$ & & $x$ \\
\hline [7] & $x$ & $\times$ & $\times$ & & $\times$ & & & & $\times$ & & $x$ \\
\hline$[8,9]$ & & & $x$ & & & & & & & & \\
\hline$[10]$ & & & $\times$ & & & & & & & & \\
\hline [11] & $x$ & & $x$ & & & & & & & & \\
\hline [12] & $\times$ & & $\times$ & & & & & & & & \\
\hline$[13,36-38]$ & $x$ & $x$ & $\times$ & $x$ & $\times$ & & $x$ & $\times$ & $\times$ & $x$ & $x$ \\
\hline$[14]$ & $x$ & & $\times$ & & & & & & & & \\
\hline [15] & $x$ & & $\times$ & & & & & & & & \\
\hline [16] & $x$ & & $\times$ & & & & & & $x$ & & \\
\hline [17] & $x$ & & $\times$ & & & & & & $\times$ & & $\times$ \\
\hline [18] & $x$ & & $\times$ & & & & & $\times$ & $\times$ & $\times$ & $\times$ \\
\hline [19] & $\times$ & & $\times$ & & & & & $\times$ & & & $\times$ \\
\hline [20] & & & & $x$ & & & & & $x$ & & $x$ \\
\hline [21] & $x$ & $x$ & $\times$ & & & & & & $x$ & & $\times$ \\
\hline [22-24] & $x$ & $x$ & $\times$ & & & & & & & & \\
\hline [25] & & & $\times$ & & & & & $\times$ & $x$ & $x$ & $\times$ \\
\hline [26] & $\times$ & $x$ & $\times$ & & & & & & & & \\
\hline [27] & $\times$ & & & & & & & & & & \\
\hline [28] & & & & $x$ & & & & & & & $\times$ \\
\hline [29] & & & $\times$ & $x$ & $\times$ & & & & $x$ & & \\
\hline$[30,31]$ & & & $\times$ & $x$ & & & & & & & \\
\hline [32] & & & & & & & $x$ & & & & \\
\hline [33] & & & & $x$ & & & & $\times$ & & $x$ & \\
\hline [34] & & & & & & & & $\times$ & $x$ & $x$ & $\times$ \\
\hline
\end{tabular}

into the workpiece and breaks if the cutting process is not stopped. Assuming this kind of wear progress gives reason to monitor the drift forces. In a series of tests [21] no consistent change of feed force or torque was observed but a certain change in the drift forces was recorded. This is again explained to be because first the cutting edge on one side and then on the other side wears.

The measurement of thrust force and torque have been linked to the waviness of the hole surface and especially the effect of tool wear to the waviness has been studied in [17]. In the analysis more emphasis has been given to thrust force than to the torque, i.e. thrust has been considered a more reliable indicator of tool wear.

Torque, feed force and strain of the table in two directions have been measured in [22-24]. The strain measurements actually in their function correspond to the measurement of drift forces, i.e. they serve the same purpose. Strain has also been measured in [25], but in this case located in the spindle and corresponding to the measurement of thrust force. Torque, drift and feed force have been also measured in [3] and compared with the measurement of ultrasonic vibration. Also in [26] torque, drift and feed force have been measured simultaneously when comparing two different types of coatings (titanium nitride and zirconium nitride).

A new method for measuring torque is suggested in [27]. The technique is based on the measurement of eddy current. The sensor can be positioned some $0.2-0.5 \mathrm{~mm}$ from the drill shank. This technique is affected by the distance between the sensor and the drill shank and also the material of the drill has an effect on the measured torque. The method is suitable for both static and dynamic torque measurements and consequently suited for both wear and failure monitoring. The method has been patented in Germany.

Based on the tests with copper alloy and a model described in [19], formulas that define the thrust force and torque as a function of feed per revolution, drill diameter and flank wear have been developed and their applicability has also been tested [6]. It should be noted that the tests indicated that the increase in cutting speed over the range studied had no significant effect on work material strength, and hence it has no significant effect on cutting forces [6]. In fact the correlation of the regression formulas with the test data without the 
rotational speed of the spindle is very good (for feed force $R^{2}=0.94$ and for torque $\mathrm{R}^{2}=0.97$ ). It is concluded that tool wear can be properly estimated knowing the thrust force and other cutting parameters, especially for larger tool wear.

Based on tests with different workpiece material hardness, formulas for torque and thrust force have been developed as a function of Brinell hardness of work material, diameter of the drill, feed per revolution, average flank wear and radius at the cutting edge [19]. It is concluded that the variation in drill life is significantly influenced by the workpiece hardness. It is speculated that it could be so that the presence of a few random workpieces with a high hardness may influence the drill life much more than a large number of workpieces with a low hardness. Hence, in an industrial operation, drills may fail very early or after a long time, depending on the occurrence of a few workpieces with a high hardness. This could explain the large variation in drill life observed in industrial conditions. The workpiece hardness also influences the amplitudes of thrust forces and torque occurring in a drilling operation. If the variation in thrust force, on account of changes in flank wear, is to be significant, the variation in workpiece hardness has to be held within $5 \%$ of the mean hardness value in order to be able to base the diagnosis of flank wear on the amplitude of thrust force or torque. This is very difficult to achieve in industrial castings. Hence, torque or thrust measurements for monitoring drill wear should be attempted only after a very close tolerance has been obtained in the workpiece hardness.

\subsection{Vibration and sound}

Vibration is widely used for condition monitoring of rotating machinery. However, vibration has not been used to the same extent in tool condition monitoring, probably because as a method it is rather sensitive to noise which is present in cutting processes. The advantages of vibration measurement include ease of implementation and the fact that no modifications to the machine tool or the workpiece fixture are required [20]. However, the disadvantages reported in the literature include dependency of the vibration signals on workpiece material, cutting conditions and machine structure.

The work of [20] deals with the development of vibration-based monitoring methods for detecting breakage of small size drills (3 $\mathrm{mm}$ diameter) and wear of larger size drills ( $6 \mathrm{~mm}$ diameter). Vibration is measured both in the transverse and axial direction. The vibration signals are considered to contain reliable features for monitoring drill wear and breakage for the following reasons: the vibrating drill length in the transverse and axial modes does not change during drilling, thus maintaining a rather constant mode frequency; the natural frequencies of the transverse and axial modes of the work- piece-drill system are basically insensitive to drill crosssectional size, thus simplifying monitoring for a wide range of drill sizes; vibrations in the directions $Y$ and $Z$ are influenced by the torque and thrust force which are the major excitation sources in drilling.

In the tests reported in [28] three accelerometers were used each measuring in the direction of one of the three axes. In [29] both vibration and the use of sound measurements are discussed. The sound measurement and analysis is discussed in more detail in [7].

Vibration measurement together with thrust force has been used in the tests reported in [30,31]. The purpose of the tests has been to obtain signal for the development of a diagnosis tool capable of recognizing tool wear. In the tests tool wear has been recorded with a vision system.

In theory, sound measurements could be expected to give the same information as can be detected using vibration measurements because in the structural boundary the mechanical vibration of the structure or tool/workpiece contact is partly transferred to airborne vibration, i.e. sound. However, quite a number of factors influence how the mechanical vibration is transferred and how it takes place at the different frequencies. Also there is a great difference when the influence of disturbances from outside sources are compared in vibration and sound measurements. The sound measurements are more vulnerable than vibration but at the same time it should be remembered that the operators sometimes or perhaps actually rather often rely on what they hear when they define whether the tool is worn or not. In [13] both vibration and sound measurements together with a number of other methods have been tested and compared in drilling, with the result that vibration was the most effective method of all of the tested methods.

A higher frequency range from 0.5 to $40 \mathrm{kHz}$ for vibration measurements has been tested with very thin drills. The reason for looking at this kind of frequency range is that the rotational natural frequencies fall into that range since for a drill of $1 \mathrm{~mm}$ diameter the natural frequency could be about $25 \mathrm{kHz}$ and for a drill of 3 mm diameter it could be about $7 \mathrm{kHz}$ [5]. In the reported examples the band-pass filtered vibration signal has given more clear indication of both tool wear and failure than the feed force signal [5].

\subsection{Acoustic emission and ultrasonic vibration}

The use of ultrasonic vibrations (UEs) in the frequency range from 20 to $80 \mathrm{kHz}$ for tool breakage detection in various metal cutting processes including drilling has been tested [4]. The practicality of using ultrasonic vibrations is explained when compared to other vibration techniques. Acoustic emission (AE) is seen to suffer from severe attenuation and multi-path distortion caused by bolted joints commonly found in machine tool struc- 
tures and restricting the mounting location of the $\mathrm{AE}$ transducer to somewhere very near the tool or workpiece. The lower frequency signal used for UE analysis does not suffer such severe attenuation and distortion, and so the transducer can be placed fairly far from the chip forming zone. In the low vibration frequency range, i.e. below $20 \mathrm{kHz}$, structural modes are prominent. A common strategy is to compare the amplitudes of several frequency bands in this range. Particular variation in the relative strengths of vibration in these bands indicate process abnormalities such as tool breakage or tool wear. This method shares the advantage of remote transducer placement with the UE method but unfortunately is much more sensitive to machine and tooling variations. Since structural modes change in complex ways with machine movement, loading, temperature, and tooling, this approach generally must be tuned empirically each time that the process is changed. In contrast, in the frequency range used for UE analysis the structural modes are so closely spaced that they form a so-called pseudocontinuum. There are no individual resonances to shift out of the analysis band with machine movement, loading, and so on.

The applicability of ultrasonic vibration measurement for the tool wear and failure detection has also been studied in [3]. In the reference the frequency range in question, i.e. from 10 to $70 \mathrm{kHz}$, is defined as acoustic emission and the used sensor with non-linear frequency response is considered as an AE-sensor. However, following Ref. [4], the frequency range in question is in this context defined as the ultrasonic range. In [3] ultrasonic vibration is compared with torque, feed and drift force measurement and proven to be a more effective means for tool wear and failure detection in drilling. The same sensor has also been used for measurements in the frequency range from 1 to $5 \mathrm{kHz}$ which normally is considered mechanical vibration.

Acoustic emission is a phenomenon which occurs when, for different reasons, a small surface displacement of a material surface is produced [32]. This occurs due to stress waves generated when there is a rapid release of energy in a material, or on its surface.

Acoustic emission with centre frequencies of 200 and $800 \mathrm{kHz}$ and also in a broader band from 100 to 1000 $\mathrm{kHz}$ has been tested in [13]. In the tests the $200 \mathrm{kHz}$ sensor was used for tool wear and the $800 \mathrm{kHz}$ sensor for tool breakage detection. The broad band sensor was used for finding the best frequency range for further investigation. Also in [32] acoustic emission was recorded in a broad band from 100 to $1000 \mathrm{kHz}$ in order to monitor tool wear.

\subsection{Spindle motor and feed drive current}

Spindle motor current is in principle a measure of the same feature as torque, i.e. they both enlighten how much power is used in the cutting process and they both also advise about the dynamics of cutting. It is fair to claim that torque is a more sensitive way to measure than is the spindle motor current since the torque sensor is located close to the cutting tool and e.g. the dynamics of the electric motor do not influence it to the same extent that they influence the current measurement. However, measuring torque is more complicated than measuring the current of the spindle motor and therefore the measurement of the current has also been widely tested and used [13,19,33,34].

Similarly, as spindle current corresponds to torque, feed drive current corresponds to the measurement of the thrust force. Again there is some similar difference in the sensitivity of the methods as described above. The feed drive current as an indicator of tool wear and failure has been studied in $[13,33,34]$.

Both feed drive and spindle current have also been measured in [25]. In these tests it has been possible to compare the measurement of feed current to the measurement of thrust force based on the use of strain gases. It is stated that typically, the strain gage is a better sensor than the feed motor current sensor for wear diagnosis. Nevertheless, the current sensor was used to investigate whether the cost effective and easily implementable current sensors alone would suffice.

The reported results in [18] for feed drive current and spindle power together with feed force and torque are quite similar. The measurement results show that all the quantities measured remain at an almost constant level during the entire tool life-time until the hole in which the drill totally fails. It is impossible to successfully apply these measurements as tool-monitoring methods, stopping the machining after the increase in one or several signals above a particular limit value before actual tool failure. However, the measurements can be used for tool-breakage detection where the machining operation is interrupted after tool breakage. With this system, one workpiece may be rejected because of the tool failure, but further damage is avoided.

\section{Signal analysis}

The kind of signal analysis methods used is of some importance. Sometimes it looks as if some researchers think that if the measured signal is acceptable then it would be possible with a clever diagnostic tool to solve everything. Unfortunately this is not the case. The diagnosis always needs to be based on reliable and meaningful information and this is where signal analysis can help by providing effective features as a basis for diagnosis. The role of signal analysis could be described as a tool which tries to pick up the meaningful information out of the mass of information. In many cases the dilemma is that the more sophisticated methods need a lot of raw 
signals and it takes time to collect this raw material and it also takes time to perform the calculations. Consequently, many of the most sophisticated methods are not suitable, e.g. for tool breakage monitoring. In addition, the results with a sophisticated analysis function are influenced by the cutting process, i.e. workpiece material, type of tool, feed rate and cutting speed which makes the diagnosis more demanding. On the other hand, very simplistic methods are fast to use and often not that sensitive to changes in cutting conditions. Unfortunately, at the same time they are not so sensitive to tool wear either. A summary of signal analysis methods that have been tested, used and reported in the literature for drill wear and failure monitoring is given in Table 2.

\subsection{Time domain signal}

The time domain signal is not very informative as such, or at least it is very time consuming to look at the raw signal in graphical format (e.g. with an oscilloscope [14]). Evaluation of the changes by measuring only the amplitude of the signal is very complicated and therefore an RMS-voltmeter is used [21]. Usually a number of statistical parameters such as root mean square (RMS), arithmetic mean, standard deviation and kurtosis are calculated and these are then used for comparison and diagnosis.

With almost all of the measuring signals the most common parameter to look at is the RMS value, which also is actually the value that is normally seen if the signal is drawn with a plotter or looked at with a voltage meter. The RMS value contains all the energy in the signal and therefore also all the noise and all the elements that depend on the cutting process. Therefore, it is not the most effective parameter but has retained its place because it is so easy to produce and understand. Besides, it does actually work when compared to other statistical parameters. In a series of tests in [13] the RMS value was compared to seven other statistical parameters, i.e. arithmetic mean, mean and standard deviation, skewness, kurtosis, maximum and minimum. The comparison showed that the RMS value is usually not the best but it is often one of the four best functioning parameters.

In the tests reported in [22-24], mean value together with the variance of the sensor signals (torque, feed and drift force) have been calculated for all of the holes. No significant changes were found in the mean and the vari-

Table 2

A summary of signal analysis methods that have been used for tool condition monitoring in drilling

\begin{tabular}{llllll}
\hline Reference number & $\begin{array}{l}\text { Time domain } \\
\text { signal }\end{array}$ & $\begin{array}{l}\text { Statistical } \\
\text { parameters }\end{array}$ & $\begin{array}{l}\text { Auto } \\
\text { regressive } \\
\text { moving } \\
\text { average }\end{array}$ & $\begin{array}{l}\text { Fast Fourier } \\
\text { transform } \\
\text { spectrum }\end{array}$ & $\begin{array}{l}\text { Cepstrum analysis } \\
\text { and }\end{array}$
\end{tabular}

\begin{tabular}{|c|c|c|c|c|c|c|c|}
\hline [1] & $x$ & $x$ & & & & & \\
\hline [3] & $x$ & $x$ & & $x$ & & & \\
\hline [4] & $x$ & $x$ & & & & & \\
\hline [5] & $x$ & $x$ & & & & & \\
\hline [6] & $\times$ & $x$ & & & & & \\
\hline$[7,27,29]$ & $\times$ & $x$ & & $\times$ & & & \\
\hline [8] & & & & & & & $x$ \\
\hline [9] & $x$ & $x$ & & & & & \\
\hline [10] & $x$ & $x$ & & $\times$ & & & \\
\hline [11] & $x$ & $x$ & & & & & \\
\hline [12] & & & & $x$ & & & \\
\hline$[13,36-38]$ & $x$ & $x$ & & $\times$ & $\times$ & & \\
\hline [14] & $x$ & $x$ & & & & & \\
\hline [15] & $x$ & $x$ & & & & & \\
\hline$[16,30,31]$ & $x$ & $x$ & & & & & \\
\hline [17] & $x$ & $\times$ & $x$ & & & & \\
\hline [18] & $x$ & $\times$ & & & & & \\
\hline [19] & $\times$ & $\times$ & & & & & \\
\hline [20] & $x$ & $x$ & & $\times$ & $\times$ & & \\
\hline [21] & $x$ & $x$ & & & & & \\
\hline$[22-24,39]$ & $x$ & $x$ & & $\times$ & & & \\
\hline [25] & $x$ & $x$ & & & & & \\
\hline [26] & $x$ & $x$ & & $\times$ & & & \\
\hline [28] & & & & $\times$ & & $\times$ & \\
\hline [32] & $x$ & $x$ & & & & & \\
\hline [33] & $x$ & $x$ & & & & & \\
\hline [34] & & & & & & & $x$ \\
\hline
\end{tabular}


ance of sensor signals. Therefore, it has been concluded that the force sensor signals in the time domain do not show any correlation with drill wear.

Based on the comparison of static and dynamic components of the feed force and torque, the analysis of the process-dynamics in drilling is considered essentially a more delicate instrument to the investigation of the wear condition than the interpretation of the increase in static feed force and torque [10].

Due to the great variation in measured signals, i.e. dynamic behaviour, average values for longer test period are often used in statistical studies. For example, in [6] average values of thrust force and torque are used when developing tool wear models.

Average, peak, RMS values and the area of thrust and torque have been used as input features in the diagnostic system described in [16]. These features have been chosen because of their previous successful application for on-line monitoring and diagnosis. Furthermore, these features were justified from the researchers' experimental observations.

Mean, peak and standard deviation have been used in the analysis of thrust force and torque signals in [17]. Of the tested statistical parameters, standard deviation proved to be the best indicator of tool wear and it was the indicator that is more closely related to the change in the standard deviation of the hole surface in composite material.

Mean, standard deviation and maximum values of the thrust force have been studied in [1]. From a series of drilling experiments conducted in the laboratory, the gradient of the thrust force has been identified to be a suitable process parameter for prediction of drill failure. A Finite Impulse Response filter using a Hamming window has been designed and used to determine the gradient of the thrust force data. Experimental evidence emphasizes the correlation between thrust force and outer corner wear; it is suggested that the sharp spikes in the thrust force that are observed under failure conditions are caused by a macroscopic stick-slip phenomenon. It has been shown that the proposed approach does not require considerable tuning for operation under a wide range of cutting conditions. This would make it ideally suited for an industrial environment.

Mean value of cutting forces (torque, drift and thrust force) has been studied in [26]. Also the maximum and minimum deviations about the mean value have been studied. In the tests two different types of drill coatings were used. The mean values were much smaller with one of the coatings (zirconium nitride) than the other (titanium nitride). The recorded mean values and deviation from these values have not given a logical indication of tool wear or alarm for tool breakage.

Smoothed average and standard deviation values of thrust force have been calculated in [9] for the detection of poor operation conditions (just before breakage, breakage, and drilling with broken tool) in micro-drilling. The processing of the data is done in four segments during each drilling cycle. These studies indicated that the average force and standard deviation value must be presented together when used as input to a neural network. Also, the study indicated that the main cause for failure was not related to tool wear. Most of the time, the very thin shaft of the drill could not carry the loads and it broke. In the test cases, total drill life varied between 0.1 and $10 \mathrm{~mm}$. There was no considerable difference between the force characteristics after the first and the 25th hole, except when the tool was broken or damaged.

A mix of statistical parameters is used in [25]. For spindle motor current the use of RMS has been justified in the following way. The low frequency energy of the spindle motor current is directly proportional to the cutting torque exerted by the tool on the workpiece. As the tool wears, the torque requirement increases and correspondingly the spindle motor current also increases. The RMS value of the spindle motor current thus becomes a valuable feature for wear prediction. In addition to the RMS value, the change in RMS value with respect to the first hole is also another good feature, since it indicates the temporal trend of the cutting torque. Also in the case of feed, motor current RMS value with a corresponding parameter indicating the change are used. For thrust force (strain gage) measurement the mean value again together with the corresponding indicator for the trend are used.

In [3] the emphasis is on the way the wear influences ultrasonic vibration in different frequency ranges, i.e. 10-20, 20-30, 30-40, 40-50, 50-60 and 60-70 kHz. The RMS value of the band passed signal has been used. There is variation in how well tool wear is observed in the different frequency ranges, although all the time the percentage increase in RMS value of some of the frequency ranges of ultrasonic vibration are always higher than is the case with the measured forces. An acoustic emission sensor in the frequency range from 1 to $5 \mathrm{kHz}$ (normally considered vibration) has proven to be especially suitable for tool wear monitoring. Apparently there have been structural vibration modes that have their frequency in this frequency range and thus increase the signal level. Tool failure has also been clearly detected with the same sensor, though the indication is clearer at higher frequencies, e.g. from 20 to $40 \mathrm{kHz}$.

Maximum stable values are used for feed force, torque, spindle and feed drive current [18]. In the case of the spindle power and current of the $Z$-axis motor, the values represent the difference in the measured quantity between cutting and idle running at the corresponding rotational frequencies.

Kurtosis value is defined as the fourth central moment of a Gaussian distribution and is a measure of peakedness of the signal. Therefore, in [20], a lot of emphasis 
is given for this value to be used as a possible indicator for tool failure. In [20] a new parameter called ratio of the absolute mean value (RAMV) is also introduced, since kurtosis was not reliable alone due to its tendency to decrease when the number of peaks in the signal becomes high. RAMV represents the ratio of absolute mean value at the current revolution of the spindle to the absolute mean value in the beginning of the drilling process, i.e. RAMV is a normalized mean value calculated with a time constant of one revolution. The RAMV value has been used with good success for triggering of the calculation of kurtosis value together with cepstrum analysis. In the tests [20] kurtosis value was found to be insensitive to cutting conditions or changes in the workpiece hardness.

One way to further process the time domain signal is to use envelope detection. As such envelope detection can be used as a practical alternative for analysing signal containing information at high frequencies and thus making the analysis process easier [7,29]. The possible use of moments of the probability distribution of intensities and time of occurrences is also discussed and a trend index (TI) based on these is described in [7,29]. The published TI curve [7] seems to indicate tool wear but does not as such give a clear indication of when the tool should be changed.

When the envelope of a signal is calculated the process at first also involves band-pass filtering of the signal. Low-pass, high-pass and band-pass filtering can all be regarded as time domain parameters and are often used, as for example band-pass filtering of the vibration signal from 0.5 to $40 \mathrm{kHz}$ in [5] in the case of thin drills in order to concentrate the analysis in the frequency range where the rotational natural frequency of the drill is expected to lie. The same approach has been used for both tool wear and failure detection.

Envelope detection together with the use of the flexible tool breakage algorithm is described in [4]. A fundamental quantity used in the signal analysis is the running mean. To establish an average signal that is not influenced by large pulses, a clipped running mean is computed each time through an algorithm loop. The running mean is a non-linearly weighted arithmetic average of the most recent samples. The clipping performs the nonlinear weighting by limiting the contribution of samples larger than a certain ratio times the current mean. A suspicion test compares the most recent sample to an upper and a lower level, each of which is a multiple of the current running mean. Together with some other similar tests based on statistical parameters calculated from the time domain signal, the test forms an algorithm that is capable of detecting tool breakage.

It should be noted that tool wear monitoring in drilling is a very periodic process, i.e. drilling one hole does not usually last very long. In addition it is possible to recognize certain stages in drilling when monitoring is practi- cal [32]. Usually in the process the drill first touches the work material and thereafter progressively drills it, with a given penetration rate. After the final depth is reached, the descending mechanism of the drilling machine is stopped and the drill keeps rotating but without drilling any further. A moment later the drill is retrieved from the hole which is then completed. Naturally, the measuring signals vary as a function of the drilling stage. In [32] the transient drilling stage (when the drill starts to penetrate into the workpiece) and the stage when the drill is stopping were found to be the best moments to monitor tool wear using the envelope value of acoustic emission.

\subsection{Autoregressive moving average}

Stationary stochastic process data in the form of a single, time dependent series can be mathematically modelled as an Autoregressive-Moving Average or ARMA model [17]. The modelling strategy involves fitting models in increasing order $n$ starting from 1 . The adequacy of the model may be tested using the conventional $F$-test. In condition monitoring the autoregressive parameters or their relations have often been used for diagnosis of faults or failures. In [17] the autoregressive model is based on the use of thrust force and torque signal and it has been used to define frequencies of modes that have then been used as the frequencies for which spectral density has been calculated. This technique has been called the Dynamic Data System (DDS) technique. With that it has been possible to get information of the contribution of each of the frequencies to the overall variance of the data. It is concluded that the dispersion analysis using the DDS technique shows a very strong correlation between the changes in the standard deviation of the lamination frequency (of composite material) component in the thrust and surface signals. This gives a direct indication of the change in the surface waviness and can be used to monitor the drill condition on-line for appropriate replacement of the drill.

\subsection{Fast Fourier transform}

The widely used Fast Fourier transform (FFT) provides a means to find out the frequency content of a measured signal. Assuming the wear influences the frequency contents of the measured signal, FFT then gives an inside view of this process. Many studies about the effectiveness of FFT have been reported [7]. Although the calculation of the power spectrum is a more sophisticated way of signal analysis than the calculation of many of the statistical parameters in the time domain and thus is a more powerful tool to get rid of noise and disturbances [13], it does suffer from limitations such as [20]: (a) materials such as cast iron are not homogeneous and will affect the amplitude of the vibration measured, 
which may cause false alarms; (b) tool damage in drilling produces a high level of transient vibrations (spikes) which are largely attenuated by the averaging procedure typically used in spectrum calculations and this makes it difficult to extract a discriminating feature to distinguish the change in the tool conditions; (c) non-uniform hardness of the workpiece material, built up edges, and micro-cracks can also cause false alarms by increasing the vibration amplitude. In order to decrease the adverse risks explained earlier, the trapezoid method has been used to calculate the area of the power spectrum between two frequencies in order to monitor tool wear with vibration [20].

The power spectral density function of torque, drift and feed force have been calculated in [26] for two different types of drill coatings tested (zirconium nitride and titanium nitride). It is concluded that the power content of the axial force and torque is significant over the entire frequency range, whereas the power content of the drift force is band limited. The power spectrum of the drift force changes from a band limited process to a wide band process when the drill is worn. The power content of the high frequencies of the cutting forces (especially the drift force component) increase as the tool approaches failure. This can be used as an index to detect the failure of the cutting tool.

Sometimes the number of points in the time domain is kept very small compared to typical values like 2048. If a small number of points is used, calculation of the power spectrum is much faster and also the frequency resolution is lower which is an advantage in the sense that even though the frequency of amplitudes in the spectrum might wander a little, they stay at the same frequency in the power spectrum. Another advantage is that the number of possible features that are used as input for a diagnosis system is in this way limited. In [12] only 256 points in the time domain have been used, which corresponds to a spectrum of 128 points in this case.

The somewhat limited 256 points in the time domain have also been used in [22-24]. The area under the power spectral density function (obtained through the Welsh method) has been studied with success. Averaging of the spectrums over a hole proved to give noisy results but this could be improved by averaging the results over a number of holes. All sensor signals, i.e. feed and drift force (strain) and torque gave similar results. Signal-to-noise analysis indicated that the power at frequencies between 50 and $300 \mathrm{~Hz}$ have the highest value of signal-to-noise ratio and, hence, are the most reliable frequencies. Comparison of the PSD plots showed that power at each frequency increases with increase in drill wear. Normalized PSD plots of all of the four sensor signals at different states of drill wear were coincident. This indicates that power at all frequencies increases proportionally with an increase in drill wear. Therefore, the change of area under the PSD plots was considered instead of power at one frequency, for integration decreases the error.

A number of FFT based functions such as autocorrelation, power spectrum (20 highest amplitudes, harmonics, as well as $1 / 3$ and $1 / 1$ octave bands), cepstrum and liftered spectrum and also two-channel functions such as cross-correlation, cross-spectrum, frequency response as well as some multi-signal frequency response function with more than two channels have been tested for tool wear monitoring in metal cutting including drilling [13]. The normal power spectrum worked well when the analysed data were fitted to a third order regression curve. Some of the two-channel functions (cross-spectrum, coherence) also proved to work well in drilling.

Cepstrum analysis is used to identify a series of harmonics or side bands in the power spectrum and to estimate their relative strength [20]. Cepstrum is calculated from the power spectrum either with inverse FFT (complex cepstrum) or taking the power spectrum of the logarithmic power spectrum (power cepstrum) [35]. In the tests [20] cepstrum analysis was performed only when a statistical RAMV indicator (explained earlier) reached a certain threshold value but the cepstrum showed larger amplitude at the frequency [35] corresponding to the time of one spindle revolution. In the tests reported in [13], cepstrum analysis also worked well in drilling and in milling. This is a logical result because both of these tool types have a number of cutting edges and when a fault starts to increase the difference between the way the cutting edges work becomes larger and consequently this is seen at the harmonics of the rotational speed of the tool which is what cepstrum then can show.

When compared to the traditional power spectrum, benefits from the use of the higher-order spectrum (HOS) features have been reported [28] in tool wear monitoring. Use of HOS features is reported to enhance monitoring performance primarily because they provide information on the strength of the non-linear and periodic component sideband structure in the received signal.

\subsection{Wavelet transform}

Wavelet transforms have become well known as useful tools for various signal processing applications [34]. Wavelet transform is described as a good solution in the time-frequency domain so that it can extract more information in the time domain at different frequency bands. Both continuous and discrete wavelet transforms are used for tool breakage detection using spindle and feed current signals. The test signals have been shown both in the time domain and after wavelet transform in [34], but no comparison with other methods is given. Hence it is difficult to compare whether what is seen clearly 
after the wavelet transform could also be seen clearly with some other analysis method, i.e. the benefits of the use of wavelet transform are not apparent.

In [8] the use of wavelet transformations together with neural networks is proposed in order to detect severe damage to micro-drills just before the breakage occurs. The use of wavelets is justified on the basis of the many weaknesses FFT has, the first being fixed resolution. The resolution of an entire frequency spectrum depends on sampling frequency and the number of data points. The second weakness is the representation of the entire spectrum, with the addition of harmonic signals, by assuming that the data window is repeated indefinitely. This assumption causes leakage problems and the transitions cannot be identified in the data window. A third weakness is the considerable noise in the transformations because of the very large degrees of freedom of the system. FFT analysis must be repeated several times and the results must be averaged to obtain smooth output. In [8], the Daubechies type wavelet system based on an orthonormal base was used. By using wavelets, the thrust force signal of the micro-drill has been simplified. The analysis indicated that the wavelet translation coefficients can represent the characteristics of micro-drilling signals with reasonable accuracy without high frequency components. The transition coefficients of all the normal micro-drills demonstrated similar patterns. The characteristics of the severely damaged micro-drills were found to be totally different. Based on these results, it is suggested that wavelets might be the perfect tool for many applications requiring automated monitoring of manufacturing operations. However, no comparison to FFT or statistical parameters has been made.

\section{Diagnostic tools}

The most simplistic methods of diagnosis in all monitoring is to use predefined limits, i.e. if a certain parameter in the analysis reaches a certain upper or lower limit this is an indication of a failure of the tool or worn tool. These types of fixed limits are often used by a human operator and they are also used in monitoring systems and form the basis of rule based expert systems. Quite similar systems based on fuzzy logic are based on these types of limits which then are fuzzy, i.e. they are not exactly defined and the limits in this case usually overlap. For example, the systems described in [33,3638] use crisp limits and the systems described in [15] use fuzzy limits.

When performing diagnosis it is often more effective to be able to follow the trend in the monitored signal and parameter than just to look at the absolute value. The reason for this is that in many cases there are outside factors affecting the absolute value. In tool wear monitoring the limits for a certain parameter, e.g. vibration amplitude, are a function of the tool type, workpiece material, cutting parameters etc. Therefore, it is more effective to follow some trend in the signal, e.g. if the amplitude has increased to double or perhaps is five times what it was when the tool was sharp it can be supposed that the tool is worn. In [20] the more sophisticated analysis is only carried out when a situation occurs that a certain parameter reaches a predefined value compared to the value in the beginning. One possible disadvantage of trend analysis is the amount of data that might need to be saved in case the whole history of the signals of the tools were to be saved. The amount of data could be enormous in the case of a machine tool with a tool magazine of tens of tools. One possible solution in order to reduce the amount of data to be saved is given in [36,37]. The suggested solution relies on the use of regression analysis and the idea is to save only the summary terms of the regression function.

The use of neural nets can be seen as an attempt to automate the process of writing the diagnostic rules, i.e. if a sufficient amount of good data exists it is possible to train a net that is capable of diagnosing the condition of the tool. In principle, neural nets can be trained to model the non-linear dependencies of the measured and analysed parameters together with process parameters. If process parameters are left out of the model, either parameters that are insensitive to cutting conditions must be used or they need to depend in such a way both on process parameters and tool wear, and failure that the model works in a number of cutting conditions. Alternatively, a number of models corresponding to the possible cutting conditions need to be developed. This is in principle the same problem or limitation as described for the rule based approach. The previous statement can be rephrased in another way, i.e. if simple models based on less sophisticated parameters are used the number of models and corresponding work increases. Unfortunately, the opposite is also true, i.e. if sophisticated models which rely on sophisticated parameters are used, the time it takes to train the models increases as does the calculation time of the parameters. A summary of approaches adopted for diagnosis of tool condition in drilling is given in Table 3 .

One of the earliest expert system concepts to monitor both the cutting process and the condition of the cutting tool is described in [33]. Among other things, the VILMOS-1 system is expected to monitor tool wear and tool breakage and also to protect the tools against overload.

In [36-38] a rule based expert system is described. The system consists of a number of modules: data acquisition and analysis, fault tree, symptom tree, rule synthesizer and fault manager. The system can be configured by the user through a graphical interface. The data are acquired through an $\mathrm{AD}$-card using a number of measuring sensors such as vibration and acoustic emission etc. 
Table 3

A summary of approaches adopted for diagnosis of tool condition in drilling

\begin{tabular}{|c|c|c|c|c|c|c|c|c|}
\hline Reference number & $\begin{array}{l}\text { Rule } \\
\text { based }\end{array}$ & $\begin{array}{l}\text { Fuzzy } \\
\text { logic }\end{array}$ & $\begin{array}{l}\text { Pattern } \\
\text { recognition }\end{array}$ & $\begin{array}{l}\text { Machining } \\
\text { influence } \\
\text { diagram model }\end{array}$ & $\begin{array}{l}\text { Multi-layer } \\
\text { perception } \\
\text { neural network }\end{array}$ & $\begin{array}{l}\text { Kohonen self- } \\
\text { organising map }\end{array}$ & $\begin{array}{l}\text { Restricted } \\
\text { Coulomb energy } \\
\text { networks }\end{array}$ & $\begin{array}{l}\text { Adaptive } \\
\text { resonance theory } \\
\text { networks }\end{array}$ \\
\hline [8] & & & & & & & & $x$ \\
\hline [9] & & & & & & & $\times$ & \\
\hline [12] & & & & & & $x$ & & \\
\hline [15] & & $x$ & & & & & & \\
\hline$[16]$ & & & & & $x$ & & & \\
\hline$[22,23,39]$ & & & $x$ & & $\times$ & & & \\
\hline [25] & & & & $x$ & & & & \\
\hline$[30]$ & & & & & $x$ & & & \\
\hline [31] & & & $x$ & & & & & \\
\hline$[33]$ & $\times$ & & & & & & & \\
\hline$[36-38]$ & $\times$ & & & & & & & \\
\hline
\end{tabular}

Signal analysis is based on the use of statistical parameters and FFT based functions. All the data are saved in a database. The actual rules of the system are written automatically through the use of fault and symptom tree modules. The idea has been to make the system very flexible so it could be used for monitoring all kinds of machining processes with all kinds of tools. The actual diagnosis of the so-called fault manager relies on a number of parameters from a number of sensors.

A generalized Machining Influence Diagram (MID) is formulated for modelling different modes of failure in drilling [25]. A faster algorithm for this model is developed to solve the diagnostic problem in real-time applications. The MID model is utilized for diagnosing two failure modes: the drill wear, and the drill failure prediction. Each drilling operation is categorized deterministically using the machining parameters. The estimation of probability that the tool is worn is done by fusing information about wear from the two sensors: spindle and feed motor current. No sensor fusion is used for tool failure prediction since only the strain gage signal is used. The state of the drill is diagnosed. It consists of three states, "ok", "worn" and "about-to-fail". Three options are available for control: "continue", "reduce feed" and "replace tool". The cost of machining is a function of the control options and the state of the drill. The response time of the on-line system is well within the desired response time of actual production lines. The instance of diagnosis is reasonably close to the actual instance of wear. The accuracy of prediction has also been significantly promising. In cases where the drill wear is not diagnosed, the system is reported to at least predict drill failure, and vice versa. Consequently, by diagnosing at least one of the two failure modes, the system is able to prevent any abrupt failure of the drill.

The system described in [15] has two input features which are the feed force and torque and the wear of the drill is clustered in four wear states, i.e. initial, small, normal and severe. The approach is fuzzy, i.e. fuzzy lim- its are defined using the fuzzy C-means algorithm. With the presented two test cases used for the development of the system the approach works well. However, the approach does not take into account the effect of the cutting process into the measured parameters, i.e. the user would need to define new fuzzy limits for different types of workpiece materials and drills and also for different cutting parameters.

A two category linear classifier has been used for the detection of drill wear [31]. Sensor fusion is used for on-line drill wear detection. The indirect indexes used are the percentage increase of the peak-to-peak amplitude of vertical acceleration and the percentage increase of the drilling thrust. A two-category linear classifier is employed to distinguish the worn-out drills from those that are still usable. Flank wear area is used to categorize the drill conditions. The wear states are classified into two categories, usable and worn-out. Based on the present data a success rate greater than $90 \%$ has been obtained for on-line detection of the drill wear in one cutting process situation.

A rather simple neural network has been developed in [30] with two input features and one output. The number of neurons in the hidden layer has been varied from four to nine. Wear has been classified into five categories, i.e. initial, slight, moderate, severe and worn-out wear (with the same data as in [31]). It is concluded that neither the percentage increase of peak-to-peak amplitude of the vertical acceleration or the percentage increase of the thrust can be used for on-line classification of drill wear. However, integrating both signals yields better results. Based on the drilling tests, a success rate of over $85 \%$ can be reached for on-line recognition of drill wear using artificial neural networks. No variation of the cutting process parameters has been included, i.e. the wider applicability of the model has not been demonstrated.

The effectiveness of artificial neural networks with different numbers of hidden layer neurons together with 
the use of adaptive activation-function slopes have been tested in the diagnosis of tool wear in [16]. In all of the models nine input features (feed force and torque based on statistical parameters with one cutting process indicator) and one output have been used. The number of neurons in the hidden layer has been varied between 14 and 22. It is concluded that modified artificial neural networks with adaptive activation-function slopes converge much faster than the conventional feed forward neural networks. Artificial neural networks can distinguish between a worn and a usable drill on-line with $100 \%$ reliability and also accurately estimate the average flank wear even under different drilling conditions. The increase in number of neurons does not necessarily improve the accuracy of on-line drill wear measurement. A neural network with $9 \times 14 \times 1$ architecture yielded the best results for on-line drill wear measurement. Although the reported results seem good even when changing the cutting conditions, the limitation in the presented material is that the variation in cutting conditions has not been documented and it is possible that there has not been any variation of feed rate in the tests.

The use of neural networks in the sensor integration has been studied in $[22,23,39]$ based on torque, feed and drift force signals. In the thesis [22] it is shown that there is no point in trying to integrate the information from these signals because they all have equally good correlation with tool wear and one sensor is adequate for monitoring and controlling tool wear. It is also stated that integration of the sensor signals can introduce redundancy in the sensor integration technique and, in the presence of noise, result in the deterioration of the estimation of drill wear. Periodograms of sensor signals at different states of drill wear are mixed and therefore it is difficult to apply the clustering technique.

A self-organizing neural network has been used in the development of a diagnosis system based on the use of feed force and torque together with FFT based feature extraction [12]. The approach is regarded as a promising empirical modeller. The conclusion is made that a certain number of feature vector components or dimensionality of a dynamic system does exist by which the drilling process can be properly characterized. Also the classification error is studied with different numbers of features. The effect of the cutting parameters is not covered in this context.

The Restricted Coulomb Energy (RCE) network is a parallel neural network modelled after the human learning and classification process [9]. The architecture of the RCE network is a feed-forward arrangement. This allows the network to classify pattern signals in real time without any special hardware. The network is composed of three layers of cells: the input layer, the internal (hidden) layer, and the output layer. The feature vectors of the pattern to be learned are presented to the input layer. The nodes of the input layer are connected to every node of the internal layer. The nodes in the internal layer are connected selectively to the output nodes during the training process. The output nodes correspond to different pattern classes. The internal connections occur in such a way that the correct output cell will be fired when an appropriate pattern class is given to the system. RCE networks use two learning mechanisms. When new patterns are presented to the network, the response of the neural network is tested without any modification of the weight matrix. If the classification of the network matches the required output, the weight matrix is not changed. Otherwise, the second method is used and the influence of the exiting nodes are modified and/or a new node will be created. In the case of breakage detection in micro-drilling, eight input features (four average, four standard deviation) based on thrust force have been used. The RCE network correctly recognised normal and tool failure cases with a higher than $90 \%$ accuracy.

Adaptive resonance networks have been tested for the detection of severe micro-drill damage just before a complete tip breakage occurs [8]. According to adaptive resonance theory (ART), adaptive resonance occurs when the input to a network and the feedback expectancies match. ART2-type neural networks have been developed to realize a self-organized stable pattern recognition capability in real time. The ART2-type neural networks compare a given input with previously encountered patterns. If the input is similar to any of the patterns, it will be placed in the same category with similar patterns. On the other hand, if the input is not similar to any of the previously presented patterns a new category will be assigned to the given input. The sensitivity of the neural network is adjusted with the vigilance value. Two approaches have been tested: in the first, 22 wavelet coefficients, and in the second, six parameters were calculated from the original 24 coefficients to represent the information of the wavelet coefficients to the neural net. The direct encoding method with 22 coefficients was found to be slower but more reliable. The ART2-type neural networks required two to three times more computational time to classify the 22 wavelet coefficients than the six parameters of the secondary encoding method. However, there was only one classification error in 61 cases. The ART2 worked much faster with the parameters of the secondary encoding; but there were at least three estimation errors in any studied case.

\section{Concluding remarks}

A summary of the monitoring methods, signal analysis and diagnostic techniques for tool wear and failure monitoring in drilling has been given. In this context only indirect monitoring methods such as force, vibration and current measurements have been covered, i.e. direct monitoring methods based on dimensional measurement 
etc. are not included. In signal analysis statistical parameters calculated from the time domain signal are widely used. Fast Fourier and Wavelet Transform are more sophisticated means of signal analysis that have also been used for tool wear and breakage detection by a number of research groups. Only a limited number of automatic diagnostic tools have been developed for diagnosis of the condition of the tool in drilling. All of these rather diverse approaches that have been available in the literature are covered in this study. In the reported material there are both success stories and attempts that have not been so successful. Only in a few of the papers have attempts been made to compare the chosen approach with other methods, i.e. many of the papers only present one approach and unfortunately quite often the test material the study is based on is limited, especially when it comes to the cutting process parameter variation, i.e. variation of cutting speed, feed rate, drill diameter and material and also workpiece material.

\section{References}

[1] A. Thangaraj, P.K. Wright, Computer-assisted prediction of drillfailure using in-process measurements of thrust force, Journal of Engineering for Industry, Transactions of the ASME 110 (1988) 192-200.

[2] Y. Kavaratzis, J.D. Maiden, System for real time process monitoring and adaptive control during CNC deep hole drilling, in: Proceedings of Comadem '89, Kogan Page, London, UK, 1989, pp. 148-152.

[3] U. Schehl, Werkzeugüberwachung mit Acoustic-Emission beim Drehen, Fräsen und Bohren, Aachen, 1991.

[4] S.R. Hayashi, C.E. Thomas, D.G. Wildes, Tool break detection by monitoring ultrasonic vibrations, Annals of the CIRP 37 (1) (1988) 61-64.

[5] K. Kutzner, U. Schehl, Werkzeugüberwachung von Bohrern kleinen Durchmessers mit Körperschallsensoren, Industrie Anzeigner 110 (82) (1988) 32-33.

[6] S.C. Lin, C.J. Ting, Tool wear monitoring in drilling using force signals, Wear 180 (1-2) (1995) 53-60.

[7] S. Braun, E. Lenz, C.L. Wu, Signature analysis applied to drilling, Journal Mechanical Design, Transactions of the ASME 104 (1982) 268-276.

[8] I.N. Tansel, C. Mekdeci, O. Rodriguez, B. Uragun, Monitoring drill conditions with wavelet based encoding and neural network, International Journal of Machine Tools \& Manufacture 33 (4) (1993) 559-575.

[9] I.N. Tansel, O. Rodriguez, C. Mekdeci, Detection of tool breakage in microdrilling operation with RCE neural networks, PED, ASME 47 (1) (1992) 83-88.

[10] C. Von Nedeß, T. Himburg, Automatisierte Überwachung des Bohrens, VDI-Z, Bd 128 (17) (1986) 651-657.

[11] K. Christoffel, W. Jung, Überwachungseinheit für die Bohrbearbeitung, Industrie Anzeigner 103 (62) (1981) 198-199.

[12] E. Govekar, I. Grabec, Self-organizing neural network application to drill wear classification, Journal of Engineering for Industry, Transactions of the ASME 116 (3) (1994) 233-238.

[13] E. Jantunen, H. Jokinen, Automated On-line Diagnosis of Cutting Tool Condition (Second version), International Journal of Flexible Automation and Integrated Manufacturing 4 (3-4) (1996) 273-287.
[14] W. König, K. Christoffel, Sensoren für die Bohrbearbeitung, Industrie Anzeigner 103 (100) (1980) 29-33.

[15] P.G. Li, S.M. Wu, Monitoring drilling wear states by a fuzzy pattern recognition technique, Journal of Engineering for Industry, Transactions of the ASME 110 (2) (1988) 297-300.

[16] T.I. Liu, K.S. Anantharaman, Intelligent classification and measurement of drill wear, Journal of Engineering for Industry, Transactions of the ASME 116 (1994) 392-397.

[17] T. Radhakrishnan, S.M. Wu, On-line hole quality evaluation for drilling composite material using dynamic data, Journal of Engineering for Industry, Transactions of the ASME 103 (1981) $119-125$.

[18] M. Routio, M. Säynätjoki, Tool wear and failure in the drilling of stainless steel, Journal of Materials Processing Technology 52 (1) (1995) 35-43.

[19] K. Subramanian, N.H. Cook, Sensing of drill wear and prediction of drill life (I), Journal of Engineering for Industry, Transactions of the ASME 101 (1977) 295-301.

[20] T.I. El-Wardany, D. Gao, M.A. Elbestawi, Tool condition monitoring in drilling using vibration signature analysis, International Journal of Machine Tools \& Manufacture 36 (6) (1996) 687-711.

[21] E. Lenz, J.E. Mayer, D.G. Lee, Investigation in drilling, Annals of the CIRP 27 (1) (1978) 49-53.

[22] A. Noori-Khajavi, Frequency and time domain analyses of sensor signals in a drilling process and their correlation with drill wear, PhD Thesis, Oklahoma State University, Stillwater, OK, 1992.

[23] A. Noori-Khajavi, R. Komanduri, On multisensor approach to drill wear monitoring, Annals of the CIRP 42 (1) (1993) 71-74.

[24] A. Noori-Khajavi, R. Komanduri, Frequency and time domain analyses of sensor signals in drilling, part 1: correlation with drill wear, International Journal of Machine Tools and Manufacture 35 (6) (1995) 775-793.

[25] K. Ramamurthi, C.L. Hough Jr., Intelligent real-time predictive diagnostics for cutting tools and supervisory control of machining operations, Journal of Engineering for Industry, Transactions of the ASME 115 (1993) 268-277.

[26] M. Valikhani, S. Chandrashekhar, An experimental investigation into the comparison of the performance characteristics of TiN an $\mathrm{ZrN}$ coatings on split point drill using the static and stochastic models of the force system as a signature, The International Journal of Advanced Manufacturing Technology 2 (1) (1987) 75-106.

[27] E. Brinksmeier, Prediction of tool fracture in drilling, Annals of the CIRP 39 (10) (1990) 97-100.

[28] R.W. Barker, G.A. Kluthe, M.J. Hinich, Monitoring rotating tool wear using higher-order spectral features, Journal of Engineering for Industry, Transactions of the ASME 115 (1993) 23-29.

[29] S. Braun, E. Lenz, Machine Tool Wear Monitoring, Mechanical Signature Analysis, Theory and Applications, pp. 321-342, Academic Press Ltd, 1986.

[30] T.I. Liu, E.J. Ko, On-line recognition of drill wear via artificial neural networks, monitoring and control for manufacturing processes, PED, ASME 44 (1990) 101-110.

[31] T.I. Liu, S.M. Wu, On-line detection of drill wear, Journal of Engineering for Industry, Transactions of the ASME 112 (1990) 299-302.

[32] A.L. Quadro, J.R.T. Branco, Analysis of the acoustic emission during drilling test, Surface \& Coating Technology 94-95 (1-3) (1997) 691-695.

[33] F. Erdélyi, C. Sántha, Monitoring tasks on boring and milling production cells, Computers in Industry 7 (1986) 65-71.

[34] X. Li, On-line detection of the breakage of small diameter drills using current signature wavelet transform, International Journal of Machine Tools \& Manufacture 39 (1) (1999) 157-164.

[35] R.B. Randall, Application of B\&K Equipment to Frequency Analysis, ISBN 878735514 0, Brüel \& Kjær, 1977.

[36] E. Jantunen, A solution for tool wear diagnosis, in: Proceedings 
of Comadem'99, Coxmoor Publishing Company, Oxford, UK, 1999, pp. 95-104.

[37] E. Jantunen, H. Jokinen, Reduction of data needed in an expert system for condition monitoring of FMS using regression analysis techniques, in: Proceedings of Comadem'96, ISBN 1-85075635-X, University of Sheffield, Sheffield, 1996.

[38] E. Jantunen, H. Jokinen, R. Milne, Flexible expert system for automated on-line diagnosis of tool condition, in: Integrated Monitoring Diagnostics \& Failure Prevention, Technology Showcase, 50th MFPT, Mobile, Alabama, 1996, pp. 259-268.

[39] A. Noori-Khajavi, R. Komanduri, Frequency and time domain analyses of sensor signals in drilling, part 2: investigation of the problems associated with sensor integration, International Journal of Machine Tools \& Manufacture 35 (6) (1995) 795-815. 